\title{
ESQUIZOFRENIA: CONCEITO, EPIDEMIOLOGIA E PAPEL DA ENFERMAGEM NA ADESÃO AO TRATAMENTO.
}

\author{
Wellington Pereira Lopes ${ }^{1}$; Aline Buriola ${ }^{1,2}$ \\ ${ }^{1}$ Universidade do Oeste Paulista - UNOESTE, Curso de Enfermagem, Presidente Prudente, SP. ${ }^{2}$ Universidade Estadual \\ de Maringá - UEM, Doutorado em Enfermagem, Maringá, PR. E-mail: aliburiola@bol.com.br
}

\section{RESUMO}

O presente trabalho tem como objetivo realizar um levantamento de textos nacionais que descreva o conceito a epidemiologia e o papel da enfermagem na adesão ao tratamento da esquizofrenia. Para isso foi realizado um estudo de revisão integrativa abordando os respectivos temas conceito da esquizofrenia, epidemiologia, e papel do enfermeiro na adesão ao tratamento. Foram revisadas informações apresentadas em trabalhos anteriores, nas seguintes bases de dados da Biblioteca virtual da Saúde: SCIELO e LILACS. O resultado aponta que a doença foi chamada de demência precoce posteriormente de esquizofrenia, os estudos epidemiológicos realizados ao redor do mundo apresentou uma taxa de prevalência de 0,9-11 por 1000 habitantes, deixando claro que o enfermeiro tem papel fundamental na orientação sobre a doença, medicamentos auxiliando assim a família na convivência com o paciente e reintegra-lo a sociedade diminuindo assim o preconceito e a discriminação pela falta de conhecimento relacionado à doença.

Palavras-chave: Esquizofrenia, enfermagem psiquiátrica, adesão à medicação, cooperação do paciente e assistência de enfermagem.

\begin{abstract}
This paper aims to conduct a survey on national texts that describe the concept of epidemiology and the role of nursing in supporting the treatment of schizophrenia. For that matter it was performed an integrative study review addressing the following issues: concept of schizophrenia, epidemiology and the role of nurses regarding the treatment. Information presented in previous works were reviewed in the following Health Virtual Collection databases: SCIELO and LILACS. The disease was called early dementia and then schizophrenia. Epidemiological worldwide studies showed a prevalence rate of 0.9 to 11 per 1000 inhabitants, making it clear that the nurse plays a fundamental role orienting about the disease and the related drugs thus helping the family that live with the patient and his reintegration into the society, thus reducing discrimination by the lack of knowledge about the disease.
\end{abstract}

Keywords: Schizophrenia, psychiatric nursing, adherence to treatment, patient cooperation and nursing assistance. 


\section{INTRODUÇÃO}

No século XIX, houve uma grande contribuição para o conceito de demência precoce. Emil Kraepelin no período de 1856 á 1926 fez a delineação da doença classificando segundo o modelo médico através de etiologias, sintomas, cursos e resultados comuns ${ }^{1}$. Kraepelin analisou alguns jovens que haviam desenvolvido um período psicótico e chegou a conclusão que havia ocorrido um "abalo psíquico", mas que não se tornavam dementes, e por esse motivo a doença podia ter evolução favorável se fosse tratada de forma correta na grande maioria dos $\operatorname{casos}^{1}$.

Na 5ạ edição de seu trabalho, Kraepelin incluiu a demência precoce como processo degenerativo, ele classificou dessa forma pelo fato dessa demência ter inicio no começo da vida. Segundo Kraepelin, nenhuma sequência de sintomas poderia caracterizar uma doença de forma definitiva, apenas o desfecho da doença levar ao diagnostico final ${ }^{8}$. Nesse período houve também a separação do transtorno maníaco-depressivo e da paranóide, Kraepelin fez uma distinção entre três tipos de transtornos sendo eles: hebefrênica, catatônica e paranóide ${ }^{1}$.

Bleuler entre 1857 e 1939 criou o termo esquizofrenia que significa divisão da mente, substituindo assim o termo demência precoce nas literaturas. Esse termo foi conceituado através da análise entre emoção e comportamento dos pacientes acometidos pela doença. Para comprovar sua teoria, Bleuler associou ambos e fez uma descrição dos sintomas específicos da doença dividindo em primários sendo eles: associação frouxa de ideias, ambivalência, autismo e alterações de afeto, e secundários os quais são: delírios e alucinações ${ }^{1}$.

Bleuler uniu os três primeiros subtipos clássicos criado por Kraepelin com a esquizofrenia simples, passando assim a fazer parte do grupo da esquizofrenia de Bleuler os subtipos paranóide, hebefrênico e catatônico ${ }^{1}$.

Em 1980 Crow fez a dicotomização da esquizofrenia em subtipos I e II ou positivo e negativo uma das classificações mais conhecidas nos dias atuais. Os sintomas da esquizofrenia positiva são: delírios e alucinações e da síndrome negativa são embotamento e pobreza de discurso ${ }^{1}$.

Os sintomas iniciais da esquizofrenia tendem comumente a surgir no final da adolescência ao inicio da vida adulta, podendo também ocorrer em outros períodos aleatórios os quais são: perda de energia, iniciativa e interesses, humor depressivo, isolamento, comportamento inadequado, negligência com a aparência pessoal e higiene, evoluindo para os aspectos relacionadas a doença surgindo as alucinações, delírios, transtorno de pensamento e de fala, perturbação de emoção e afeto, déficit de cognição 1 . 
As alucinações podem aparecer em algum momento no desenvolvimento da doença ocorrendo alucinações visuais que acomete cerca de $15 \%$ dos pacientes, auditivas 50\% dos doentes e táteis $5 \%$ e os delírios que acomete mais de $90 \%$ das pessoas portadoras dessa doença psiquiátricas ${ }^{1}$.

No que diz respeito ao papel do enfermeiro no tratamento aos pacientes com esquizofrenia, á partir do momento que o enfermeiro se torna atuante dando suporte ao tratamento do paciente com transtorno metal na sociedade, ele alivia a sobrecarga familiar, prevenindo assim, que outra pessoa da família adoeça pelo fato de estar sobrecarregado por cuidar de um portador de esquizofrenia, por isso é essencial que os programas de saúde mental para pacientes com transtornos psíquicos graves e persistentes atenda toda a demanda, reduzindo assim a responsabilidade familiar, além disso, é necessário que aconteça ações que propiciem momentos agradáveis de interação e recreação entre doentes e familiares para que seja fortalecido os vínculos e os laços entre eles o que ajudará na adesão e evolução do tratamento ${ }^{7}$.

Neste sentido, é possível perceber a importância do enfermeiro para o controle do medo, da fragilidade, das angústias e das dificuldades enfrentadas pelo cuidador de um paciente com transtorno mental sendo que a doença é de uma grande complexidade e que ainda não se sabe a etiologia com perfeição dessa psicopatologia. Essa pesquisa se propôs a alcançar os seguintes objetivos: realizar o levantamento e análise das publicações e suas contribuições em relação ao conceito da esquizofrenia, perfil epidemiológico e papel da enfermagem na adesão ao tratamento.

\section{METODOLOGIA}

Trata-se de uma pesquisa de revisão integrativa um método que busca a avaliação critica e a síntese de resultados de estudos e evidencias cientificas sobre um tema. Esta investigação tem como objetivo revisar a literatura apresentando conceitos gerais e produzindo assim conhecimento para futuras intervenções na assistência à saúde, identificando problemas e direcionando novos estudos e pesquisas.

Foi realizado uma busca no banco de dados SCIELO (Scientific Eletronic Library Online) LILACS (Literatura Latino Americana e do Caribe em Ciências da Saúde) no período de março a julho de 2015. Para a seleção dos trabalhos foram utilizados os seguintes unitermos: Esquizofrenia, enfermagem psiquiátrica, adesão a medicação, cooperação do paciente e assistência de enfermagem. 
Os critérios de inclusão das referências foram possuir aderência ao objetivo proposto; fazer referência ao conceito de esquizofrenia, trazer dados epidemiológicos sobre a doença, adesão ao tratamento ou papel do enfermeiro no tratamento da esquizofrenia. Vale destacar que foram usados todos os trabalhos encontrados que se encaixam nos critérios citados acima independente do ano da sua publicação.

Após a busca foram encontradas 305 produções cientificas, sendo desses apenas 9 analisados, por satisfazerem os critérios de inclusão os motivos que levaram, a não escolha dos outros foram não ter como foco especifico o conceito, epidemiologia.

O conteúdo dos artigos foi coletado, registrado e analisado individualmente, ou seja, primeiramente trabalhou-se de forma individual com cada estudo para depois analisá-los como um todo e interpretá-los. A análise crítica dos dados foi desempenhada por meio de leitura, com vista à crítica externa - crítica do texto, da autenticidade e da providência - e crítica interna - crítica do valor interno do conteúdo, do valor que representa o trabalho e as ideias nele contidas, para após proceder à reflexão do tema, fundamentada em literatura.

Ressaltando que todos os artigos selecionados são da área da saúde, os textos foram submetidos a comparações com os demais para verificar possíveis contradições entre seus autores. Em geral apresentaram informações sobre a Política de Humanização e seus benefícios, como também as ações executadas pelos Agentes de saúde e seus principais conflitos.

\section{RESULTADOS E DISCUSSÃO}

Dos nove (9) artigos selecionados, cinco (5) pertencem à base de dados SCIELO (Scientific Eletronic Library Online), e três (4) pertencem à base de dados LILACS (Literatura Latino Americana e do Caribe em Ciências da Saúde). Após a coleta de dados, e a análise crítica dos artigos foram encontrados os resultados descritos na tabela 1 e discussão. 
Tabela 1. Distribuição dos artigos encontrados para a revisão.

\begin{tabular}{c|c|c|c}
\hline Ano de Publicação & Numero de artigos & $\begin{array}{c}\text { Tema principal do } \\
\text { artigo }\end{array}$ & $\begin{array}{c}\text { Especificação do } \\
\text { estudo. }\end{array}$ \\
\hline 2000 & 2 & $\begin{array}{c}\text { Esquizofrenia entre } \\
\text { homem e mulher }\end{array}$ & Artigo de reflexão \\
\hline 2006 & 2 & Conceito esquizofrenia & Material de apoio \\
\hline 2007 & 1 & $\begin{array}{c}\text { Enfermagem e Adesão } \\
\text { ao tratamento }\end{array}$ & Artigo de reflexão \\
\hline 2011 & 2 & $\begin{array}{c}\text { Enfermagem e adesão } \\
\text { ao tratamento }\end{array}$ & Artigo de reflexão \\
\hline 2013 & 1 & $\begin{array}{c}\text { Enfermagem e Adesão } \\
\text { ao tratamento }\end{array}$ & Artigo de reflexão \\
\hline & & $\begin{array}{c}\text { Diagnostico de } \\
\text { esquizofrenia }\end{array}$ & Artigo de reflexão \\
\hline
\end{tabular}

Estudos epidemiológicos realizados ao redor do mundo apresentou uma taxa de prevalência de 0,9-11 por 1000 habitantes, entretanto a incidência em esquizofrenia é um pouco menor sendo 0,1-0,7 novos casos a cada 1000 habitantes $^{1}$.

Existe também uma discussão em relação ao inicio da doença entre o homem e a mulher, em geral nos homens os sintomas tendem a aparecer entre 18-25 anos e as mulheres em torno de 25-35 anos. No inicio da adolescência o índice entre homem e mulher é 2:1, entretanto quando são avaliados os pacientes acima de 50 anos esse índice percentual tende a se inverter sendo que aproximadamente $3 \%$ a $10 \%$ das mulheres desenvolvem a doença após os 45 anos $^{9}$.

Estudos mostram que existe uma evolução maior em mulheres que fazem o tratamento da doença do que nos homens, isso pode ser devido à personalidade pré-mórbida comprometida, adaptação social e números de suicídios ${ }^{9}$.

A causa da esquizofrenia ainda é desconhecida, entretanto alguns fatores de risco como hereditariedade e a variação entre diversos tipos de culturas podem ser alguns fatores relacionados à etiologia da doença. Se houver um parente com esquizofrenia existe uma grande probabilidade de desenvolver a doença, por outro lado vale também destacar que o ambiente de convivência pode ser um fator de risco para desencadear esse transtorno psicótico ${ }^{1}$.

Alguns estudos nas ultimas décadas trazem a hipótese de que a doença pode estar relacionada ao desenvolvimento do sistema cerebral durante a vida intrauterina e a eventos que podem ter acontecido de forma precoce logo após o nascimento, os quais de alguma forma interferiram no desenvolvimento no sistema neural do individuo. A falta de nutrientes para o feto, a redução do suprimento de oxigênio, ferro, iodo, glicose poderiam estar relacionados aos prejuízos causados ao sistema nervoso ${ }^{1}$. 
Por ser uma condição crônica, a esquizofrenia precisa de tratamento por tempo indeterminado e constante o qual se da pelo uso de medicamentos antipsicóticos, a esquizofrenia não tem cura, entretanto, essas drogas podem amenizar os sintomas e ajudar o paciente na adaptação e no bem-estar pessoal . $^{3}$

Por outro lado, o tratamento com antipsicótico muitas vezes não tem sucesso devido a não aderência dos pacientes ao tratamento comprometendo e agravando cada vez mais o caso conforme ocorrem novas recaídas e novos surtos psicóticos ${ }^{3}$.

Alguns fatores importantes que devem ser levados em consideração ao paciente e a doença é o reconhecimento pessoal do individuo em relação ao seu estado mental, o apoio familiar, as crenças e o meio que esse paciente está inserido ${ }^{3}$.

Em um estudo realizado foi identificado que algumas barreiras podem atrapalhar a evolução do tratamento sendo elas: insatisfação com a terapia instituída, dificuldade no manejo dos medicamentos, crença de que a medicação pode the causar mal ou de que o mesmo é ineficaz, dificuldade na aquisição de conseguir a receita médica, falta de informação a cerca do transtorno e efeitos colaterais ${ }^{3}$.

A maioria dos estudos sobre a adesão ao tratamento em esquizofrenia foi realizado em países desenvolvidos, já nos países em desenvolvimento existe muito pouco estudo sobre o assunto, o que torna difícil ter dados claros e objetivo sobre essa questão, porém os dados encontrados revelam que a falta de adesão ao tratamento nesses países desenvolvidos muitas vezes acontece devido a falta de apoio social, efeitos colaterais das medicações, comportamentos violentes, tentativas de suicídios situação de rua entre vários outros. Por outro lado, no Brasil os poucos estudos sobre o a adesão não usa técnicas diretas de avaliação, mas os fatores encontrados pela falta de adesão é praticamente os mesmos relatados nos países desenvolvidos ${ }^{4}$.

Devido à falta de entendimento por parte dos familiares e até mesmo devido às dificuldades acarretadas pelos delírios presentes na esquizofrenia, à adesão ao tratamento ${ }^{5}$ nem sempre é feita da melhor forma possível fazendo com que a doença evolua e se torne cada vez mais difícil obter um resultado satisfatório, por esse motivo o enfermeiro tem um papel fundamental na analise biopsicossocial ${ }^{2}$ do portador da doença e da família que o mesmo está inserido, podendo ele intervir através de visitas familiares, acompanhamento familiar dos pacientes que estão internados nas suas enfermarias de preferência em momentos separados primeiro com a família e depois com o paciente aproveitando esse tempo para explicar um pouco mais sobre a doença e sobre a importância de conduzir os tratamentos medicamentoso e terapêutico de forma correta para evitar novas crises e assim melhorar a qualidade de vida do 
portador da doença e de seus familiares e coordenar grupos e oficinas de terapia ocupacional buscando desenvolver novas habilidades que possam contribuir de forma positiva no tratamento.

Além dessas ações, o enfermeiro deve sempre se atualizar e procurar encontrar formas de minimizar o máximo possível os efeitos colaterais dos medicamentos, visto que esse é um dos principais fatores relacionados a não adesão, partindo desse principio o profissional também precisa considerar essa possível rejeição a forma de tratamento prescrita pelo médico e por esse motivo é essencial que o enfermeiro de ênfase aos benefícios dos tratamentos, explicar os possíveis efeitos colaterais e facilitar o acesso as medicações necessárias.

\section{CONSIDERAÇÕES FINAIS}

A análise dos artigos possibilitou identificar que a esquizofrenia é uma doença que tem sido estudada a mais de um século e que a mesma passou pelo conceito de demência precoce devido ao fato da maioria dos pacientes portadores da doença ser jovens, até o conceito atual de esquizofrenia onde puderam avaliar e separar os sintomas positivos e negativos, além disso, conclui-se que os dados epidemiológicos revelam que a maioria dos sintomas da doença começa entre os 18 aos 35 anos podendo variar entre homens e mulheres.

O presente estudo trás uma contribuição satisfatória para os enfermeiros a respeito do conceito histórico sobre a esquizofrenia de alguns sintomas da doença e das possíveis intervenções que poderão ser realizadas pelos profissionais, para que assim, o enfermeiro possa atuar de forma direta na evolução do quadro clinico do paciente na reabilitação do mesmo e na convivência familiar, entretanto, pudemos observar que existem poucos estudos que descrevem os problemas familiares e as intervenções de enfermagem que foram usadas para que tal problema fosse solucionado, dessa maneira, é evidente que essa é uma área que precisa de novos estudos para contribuir com a comunidade científica e para os demais profissionais, visando melhorar a qualidade de vida do paciente e de seus familiares, além de acrescentar mais conhecimento cientifico ao nosso desenvolvimento teórico.

\section{REFERÊNCIAS}

1. Silva, RC. Esquizofrenia. Uma revisão Rev. Psicol 2006. USP São Paulo vol.17 no 4 Pp 263285. Disponivel em: $\quad$ www.scielo.br/scielo.php?script=sci arttext\&pid=S0103$\underline{65642006000400014 \& \text { Ing=en\&tlng=pt. 10.1590/S0103-65642006000400014. }}$

2. Giacon BCC, Galera SAF. Primeiro episódio da esquizofrenia e assistência de enfermagem. Rev. esc. enferm. USP [Internet]. 2006 June [cited 2015 July 11] ; 40( 2 ): 286-291. Available 
3. Nicolino PS, Vedana KGG, Miasso Al, Cardoso L, Galera SAF. Esquizofrenia: adesão ao tratamento e crenças sobre o transtorno e terapêutica medicamentosa. Rev. esc. enferm. USP [Internet]. 2011 June [cited 2015 July 11] ; 45( 3 ): 708-715. Available from: <http://www.scielo.br/scielo.php?script=sci_arttext\&pid=S0080-62342011000300023\&lng=en http://dx.doi.org/10.1590/S0080-62342011000300023

4. Silva TFC, Lovisi GM, Verdolin LD, Cavalcanti MT. Adesão ao tratamento medicamentoso em pacientes do espectro esquizofrênico: uma revisão sistemática da literatura. J. bras. psiquiatr. [Internet]. 2012 [cited 2015 July 11] ; 61( 4 ): 242-251. Available from: http://www.scielo.br/scielo.php?script=sci arttext\&pid=S004720852012000400008\&lng=en. http://dx.doi.org/10.1590/S0047-20852012000400008

5. Rosa MA, Elkis H. Adesão em esquizofrenia. Rev. psiquiatr. clín. [Internet]. 2007 [cited 2015 July 11] ; 34( Suppl 2 ): 189-192. Available from: http://www.scielo.br/scielo.php?script=sci_arttext\&pid=S0101$60832007000800008 \& \operatorname{lng}=e n$. http://dx.doi.org/10.1590/S0101-60832007000800008.

6. Rocha NBF; Queirós CL; Bravo AR; Silva ASA; Marques APS; Oliveira CR, Susana A; Pereira NGF. Análise qualitativa do impacto do programa de treino metacognitivo e da cognição social em pessoas com Esquizofreia 2013. Estudos de Psicologia (Natal), 18(4), 559-568. Retrieved July 11, 2015, from $\quad$ http://www.scielo.br/scielo.php?script=sci arttext\&pid=S1413$\underline{294 X 2013000400003 \& \operatorname{lng}=e n \& t \operatorname{lng}=\text { pt. } 10.1590 / \text { S1413-294X2013000400003 }}$

7. Macedo, TPM; Fernandes CA; Costa IS. Rede de apoio social de pacientes com diagnóstico de esquizofrenia: estudo exploratório. Estud. psicol. (Natal), Natal , v. 18, n. 4, p. 639647, Dec. 2013 . Available from http://www.scielo.br/scielo.php?script=sci arttext\&pid=S1413294X2013000400012\&lng=en\&nrm=iso

access on 11 July 2015. http://dx.doi.org/10.1590/S1413-294X2013000400012.

8. Elkis H. A evolução do conceito de esquizofrenia neste século. Rev. Bras. Psiquiatria. [Internet]. 2000 May [cited 2015 July 11] ; 22( Suppl 1 ): 23-26. Available from: http://www.scielo.br/scielo.php?script=sci arttext\&pid=S1516$\underline{44462000000500009 \& \operatorname{lng}=e n \text {. http://dx.doi.org/10.1590/S1516-44462000000500009 }}$

9. Chaves AC. Diferenças entre os sexos na esquizofrenia. Rev. Bras. Psiquiatr. [Internet]. 2000 May [cited 2015 July 11]; 22( Suppl 1 ): 21-22. Available from: http://www.scielo.br/scielo.php?script=sci arttext\&pid=S1516-

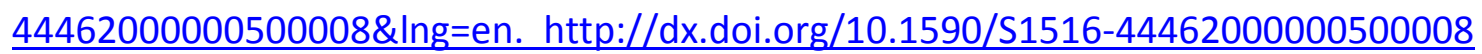

